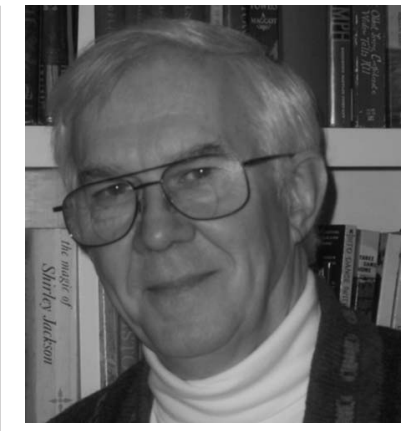

\section{Reading in the 21st Century}

The change from print to digital delivery of OE for SPIE members has caused some of us to confront a dilemma that we had managed to evade till now. When a journal arrives in the mail, we flip through it, scan the most interesting papers and, if anything concerns our current research, we dog-ear, post-it note, or copy it for closer reading or future reference. Now most of us don't receive a print journal. For some of us this is a difficult change.

Part of this is, I think, generational. Many younger persons have grown up or have been accustomed to acquiring information off televisions and computer monitors and the change is of little consequence. Having served as editor of OE since 1998, I review close to 900 manuscripts each year. While I only read a fraction of the papers, I have to look at all of them to see if the writing is sufficiently clear to be reviewed and to determine the topical area so that an Associate Editor can be assigned to conduct the review. It would be inefficient and very wasteful to print out these papers, so I spend a great deal of time reading on a computer screen. Granted, it's a $1280 \times 1024$ LCD Apple Cinema 17-in. display, which makes it easy to read, but I have to maintain a certain location and posture. Even with a decent chair and screen height, it gets tiring. Oh, for the portability of the book!

There is a devil's bargain that we have made with our technology. The devil says, "How would you like to read almost anything that is published these days?" And we answer "Wow, what a great idea! Yes, yes. Give us this torrent of information!" And the devil says, "Oh, there is one thing. I require that you sit in front of a backlighted display while you take in all this material. And in some cases, my minions will pepper you with pop-up ads, spam, and viruses, so that you do not become complacent and self-satisfied."

At some point the reader must deal with the devil. From childhood on we have enjoyed the portability and comfort of books. (Have you ever crossed your legs while reading a paper on a computer screen...even if it is attached to a laptop?) You need to read the paper at your leisure, comment on it, annotate it, and store it for future reference. You need to be able to store and retrieve the data. So you print out the paper, mark it up with a pen or highlighter, file it in folder, and stick it in a file drawer. The paper's worth is now tied to the impression that it made on you upon reading it and your file retrieval system, your memory. This may be satisfying to you at the time, but is it the optimum approach to acquiring and using information today?

One of the biggest drawbacks of your beloved bound collections of paper is that you cannot click on a paper listed in the references and have a copy of it (or, at least, its abstract) magically appear in your hand. Whereas, performing the same operation on a reference that is linked by standard Digital Object Identifier (DOI) protocol, through an organization called CrossRef, will do so. There are other great advantages to online access as well, such as searchability across all papers. Also, SPIE recently expanded its Digital Library archive, so you can now access journal and proceedings papers back to 1990. Beyond this, papers of interest can be downloaded to your computer and stored in a folder for future use. Now this is not the same as a folder in the drawer of a file cabinet. I can search on my hard disk and specify search terms and limit the search to .pdf files. On the Macintosh using Spotlight, a search feature built into the operating system, I can find those papers that contain those terms anywhere in the paper. When Microsoft Vista is released, a similar capability should become available. As a backup, your folder could be burned to a CD.

That still leaves the devilish bargain in place. However, with some effort the process can be made more agreeable. First, if you need to read the paper, print it out, take it to your hammock, and read it between snoozes. Mark it up. If you want to keep the comments in a digital format, you can transfer them to the .pdf using Adobe Acrobat Standard or, if you are on a PC, there are several programs that will attach comments to a document.

I realize that printing a paper represents a use or, some would say, a waste of resources. But for those uncomfortable with extended reading from a computer monitor, this represents a way of using your digital pipeline to get what you need. Perhaps as technology evolves, our monitors will become portable and lightweight, taking on the characteristics of books and newspapers. Till then we can use our resources to expand our awareness and knowledge in our field of research. After all, that's what this journal and others are all about.

Donald C. O'Shea Editor 